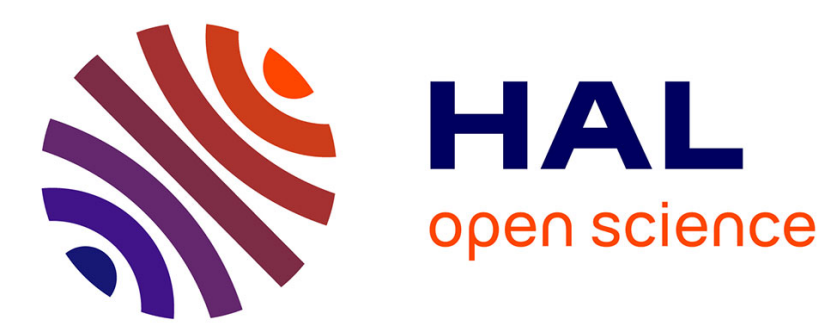

\title{
Un nouveau type de pompe à chaleur: la PAC à compression-absorption à fonctionnement alterné
}

\author{
E. Dietrich, P. Le Goff
}

\section{To cite this version:}

E. Dietrich, P. Le Goff. Un nouveau type de pompe à chaleur: la PAC à compressionabsorption à fonctionnement alterné. Revue de Physique Appliquée, 1986, 21 (1), pp.45-52. 10.1051/rphysap:0198600210104500 . jpa-00245409

\section{HAL Id: jpa-00245409 https://hal.science/jpa-00245409}

Submitted on 1 Jan 1986

HAL is a multi-disciplinary open access archive for the deposit and dissemination of scientific research documents, whether they are published or not. The documents may come from teaching and research institutions in France or abroad, or from public or private research centers.
L'archive ouverte pluridisciplinaire HAL, est destinée au dépôt et à la diffusion de documents scientifiques de niveau recherche, publiés ou non, émanant des établissements d'enseignement et de recherche français ou étrangers, des laboratoires publics ou privés. 


\title{
Un nouveau type de pompe à chaleur : la PAC à compression-absorption à fonctionnement alterné
}

\author{
E. Dietrich et P. Le Goff \\ L.S.G.C.-CNRS-ENSIC, 1, rue Grandville, 54042 Nancy Cedex, France
}

(Reçu le 10 mai 1985, accepté le 4 octobre 1985)

\begin{abstract}
Résumé. - Nous proposons un nouveau type de pompe à chaleur qui est, par certains aspects, intermédiaire entre la PAC à absorption et la PACà compression. Comme la PACà absorption, elle utilise un fluide de travail qui est un mélange binaire d'un solvant volatil et d'un soluté non volatil. Comme la PAC à compression, elle utilise de l'énergie mécanique comme énergie de haut niveau. De plus cette PAC a la particularité d'être composée d'un seul et même appareil qui joue le rôle alternativement du mélangeur et du séparateur thermo-mécaniques = d'où vient son nom

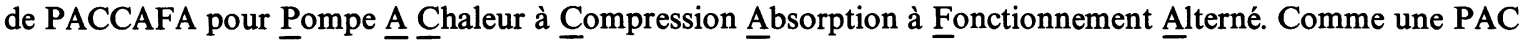
à compression, la PACCAFA est formée d'un évaporateur, d'un compresseur, d'un condenseur et d'un détendeur auxquels on a ajouté trois réservoirs de stockage respectivement pour le diluat, le concentrat et le solvant. Nous calculons la valeur maximale théorique du Coefficient de Performance d'une PACCAFA et le comparons à celui d'une PAC à compression classique. Nous montrons les avantages de la PACCAFA, dans son application au chauffage des bâtiments : suppression de la chaudière de combustion d'appoint pour périodes froides; fonctionnement du compresseur à puissance quasi constante; existence d'un stockage interne permettant une optimisation multi-critère, tenant compte des fluctuations de la température extérieure, de la demande des utilisateurs et des tarifs de l'électricité.
\end{abstract}

\begin{abstract}
The new heat pump, here proposed, is intermediate between a compression heat pump and an absorption heat pump. Like the absorption heat pump, it uses a binary mixture made of a volatile solvent and a non-volatile solute. Like the compression heat pump, it uses mechanical energy as high level energy. Furthermore this heat pump has the advantage to be composed with but one machine alternatively working as a thermo-mechanical mixer and as a thermo-mechanical separator : whence its name CAHPAW for Compression-Absorption Heat Pump Alternatively Working. Like the compression heat pump, the CAHPAW is composed with an evaporator, a compressor, a condenser and a pressure reducing valve at which three storage tanks have been added respectively for the diluted and concentrated solutions and the pure solvent. The theoretical maximum value of the coefficient of performance of a CAHPAW is calculated and is compared with that of a classical compression heat pump. The advantages of the CAHPAW for house heating are : suppression of combustion furnace for cold periods; the compressor works at a quasi-steady state, with a constant power; the existence of an internal storage permits a multi-criteria, optimization taking account of the outside temperature variations, the consumer's requirement and the electricity prices.
\end{abstract}

\section{Introduction.}

Rappelons tout d'abord que le but même de toute pompe à chaleur qu'elle soit à compression ou à absorption, est de remonter le niveau thermique d'une source de chaleur disponible à basse température, en lui apportant de l'exergie.

Dans une précédente publication (Réf. [1]) nous rappelions que l'exergie ainsi fournie au système est purement mécanique dans le cas de la PAC à compression et purement thermique dans le cas de la PAC à absorption. Nous présentions ensuite un nouveau type de pompe à chaleur intermédiaire entre les deux précédentes, en ce sens que le fluide de travail est un mélange binaire à volatilités différentes comme dans les PAC à absorption et que l'exergie fournie au séparateur est au moins en partie de l'énergie mécanique sur l'arbre d'un compresseur, comme dans une PAC à compression.

Nous proposions d'appeler ces systèmes intermédiaires des PACCA, pour " $\underline{\text { Pompe } \underline{\mathrm{A}} \text { Chaleur à }}$ Compression-Absorption ", et nous décrivions trois variantes, selon le mode d'apport de la partie d'exergie fournie sous forme thermique.

Nous proposons ici une nouvelle variante de pompe à chaleur, faisant encore partie de la "famille» des 
PACCA et caractérisée par les points suivants qui la différencie des autres variantes :

- le mélangeur comprend un compresseur et utilise donc à la fois de l'exergie mécanique et de l'exergie thermique, contrairement aux autres variantes où elle était purement thermique,

- le séparateur et le mélangeur ne constituent qu'un seul et même appareil donc avec un seul évaporateur, un seul condenseur et un seul compresseur, les fonctions séparation et mélangeage étant effectuées alternativement dans cet unique appareil.

Nous le désignerons donc par le sigle : PACCAFA, pour Pompe A Chaleur à Compression- $\underline{A}$ bsorption à Fonctionnement Alterné.

\section{Quelques rappels sur la PAC â compression clas- sique air-eau.}

Rappelons qu'une PAC à compression est classiquement composée des éléments suivants (cf. Fig. 1) :

* un évaporateur où un liquide volatil (par exemple de l'ammoniac) s'évapore au contact d'une paroi chauffée de l'extérieur, par exemple par l'atmosphère extérieure à $10^{\circ} \mathrm{C}$;

* un compresseur qui fait passer la vapeur de la pression $P_{1}$ à la pression $P_{2}$ (par exemple de 5,2 à $16 \mathrm{~atm})$

* un condenseur où la vapeur, à la pression $P_{2}$, se condense sur une paroi à $40^{\circ} \mathrm{C}$ et réchauffe ainsi un fluide caloporteur destiné à l'utilisation, par exemple au chauffage d'une habitation;

* un détendeur où le liquide repasse de la pression $P_{2}$ à la pression $P_{1}$, pour être recyclé dans l'évaporateur.

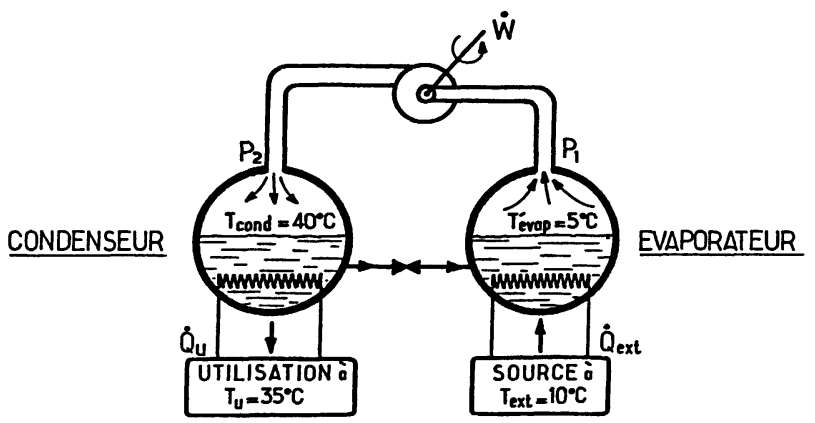

Fig. 1. - Pompe à chaleur à compression de vapeur d'un fluide pur pour chauffage d'un bâtiment.

[A vapour compression heat pump of a pure fluid for heating a building.]

Le coefficient de performance est d'autant plus grand que la différence de températeure entre évaporateur et condenseur est plus faible.

Dans le cas extrême où l'on veut produire de l'eau chaude à $60^{\circ}-80^{\circ} \mathrm{C}$, avec une température extérieure inférieure à $0^{\circ} \mathrm{C}$, le COP devient si faible que le système n'est plus rentable. C'est pourquoi on considère généralement qu'une telle PAC classique air/eau ne peut pas fonctionner seule. Il faut nécessairement lui associer une chaudière d'appoint, à combustible, pour les périodes les plus froides. Un tel surinvestissement constitue un important inconvénient de ce système bi-énergie.

\section{Le principe de la PACCAFA.}

Le système que nous proposons ci-après, basé sur les deux principes de compression et d'absorption, a au contraire l'avantage d'être mono-énergie, en ce sens qu'il fonctionne uniquement à l'électricité, même par les temps les plus froids et ne nécessite pas l'installation d'une chaudière d'appoint.

Son principe est le suivant :

On remplace le fluide pur, à savoir l'ammoniac, par un mélange binaire, composé de ce même fluide appelé «solvant volatil » et d'un soluté non volatil, par exemple le thiocyanate de sodium qui a la propriété d'abaisser fortement la pression de vapeur du solvant.

C'est exactement ce même type de mélange que l'on utilise habituellement dans les pompes à chaleur à absorption (Réf. [5]).

L'évaporateur-compresseur-condenseur fonctionne alors en régime cyclique tantôt en "séparateur " du mélange, tantôt en « re-mélangeur » des constituants du mélange.

Quand il opère en séparateur, une solution diluée (de soluté dans le solvant) est injectée en tête de l'évaporateur et on recueille une solution concentrée en pied de l'appareil, alors que du solvant pur est condensé et recueilli au condenseur (voir Fig. 2b).

Quand il opère en mélangeur, on injecte au contraire du solvant pur en tête de l'évaporateur - et de la solution concentrée en tête du condenseur. La vapeur de solvant se condense sur, et se mélange dans, cette solution - et donc la dilue. On recueille une solution diluée en pied du condenseur (voir Fig. 2a).

Trois réservoirs, respectivement pour le concentrat, le diluat et le solvant pur, sont alternativement remplis et vidés, au cours de chaque cycle de séparationmélangeage. Leurs volumes dépendent évidemment de la durée des cycles.

De plus cette PACCAFA bénéficie de la nouvelle technique de réalisation des PAC à absorption que nous avons proposée ailleurs (Réf. [2] et [5]) c'est-àdire de films ruisselants sur parois verticales qui autorisent d'excellents transferts de chaleur et de faibles coûts de fabrication.

Quand cet appareil fonctionne en séparateur, nous l'appelons VACOSEP (pour VAporiseur-COndenseur-SEParateur) - quand il opère en mélangeur, nous l'appelons VACOMEL (pour VAporiseurCOndenseur-MELangeur) (Réf. [3] et [5]).

\section{Comparaison de la PACCAFA et de la PAC clas- sique.}

Pour effectuer la séparation, il faut apporter de l'énergie au système (grâce au compresseur qui aura à vaincre 
une différence de pression plus grande qu'une pompe à chaleur à compression). Il y a conversion d'énergie mécanique en énergie chimique de séparation. Au contraire, lors du mélangeage, de l'énergie chimique est reconvertie en énergie mécanique disponible pour l'utilisateur.

En conséquence, la comparaison de cette PACCAFA avec une PAC à compression classique, opérant entre les mêmes températures d'évaporation et de condensation, montre que la PACCAFA consommerait plus d'énergie mécanique pendant les périodes de séparation et moins d'énergie pendant les périodes de mélangeage.

En fait, la décision d'opérer en séparateur ou en mélangeur dépendra essentiellement de la température extérieure : par $-15^{\circ} \mathrm{C}$, alors qu'une PAC normale devenue inopérante "économiquement", serait remplacée par la chaudière à fuel, au contraire la PACCAFA continuera à fonctionner. Pour ce faire, on la fera fonctionner en mélangeur ce qui reviendra à " aider " le compresseur en lui apportant l'énergie de mélangeage.

Par contre, en temps doux, par $+10^{\circ} \mathrm{C}$, une PAC normale consommerait très peu d'énergie mécanique.

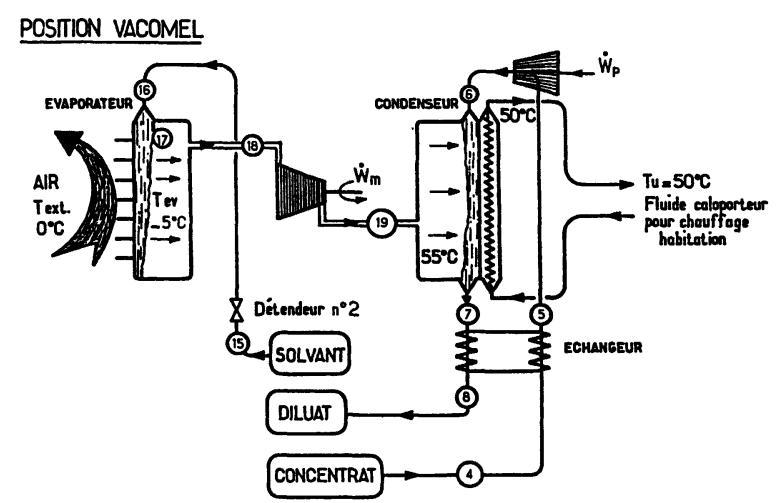

Fig. 2a. - Schéma de principe de la PACCAFA : position VACOMEL.

[Principle of the CAHPAW : VACOMIX state.]

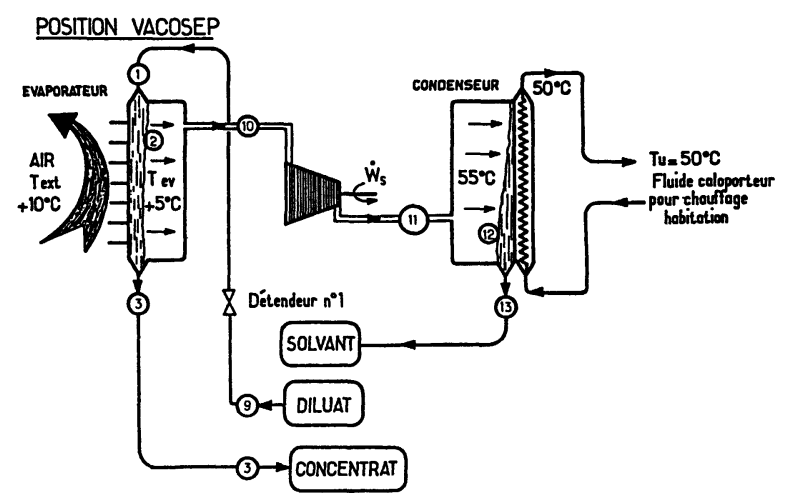

Fig. 2b. - Schéma de principe de la PACCAFA : position VACOSEP.

[Principle of the CAHPAW : VACOSEP state.]
On fera alors fonctionner la PACCA-FA en séparateur. Elle aura donc à fournir une énergie supplémentaire, celle de séparation.

Au total, en dépit des fluctuations importantes de la température extérieure et donc des fluctuations importantes de demandes d'énergie de chauffage, le compresseur aura à fournir une puissance mécanique $a ̀$ peu près constante, la séparation-mélangeage jouant le rôle de stockage d'énergie.

A la vérité, la demande de chauffage ne dépend pas seulement de la température extérieure. Elle dépend aussi évidemment de l'activité humaine : par exemple dans les bâtiments professionnels, la température imposée sera $19^{\circ} \mathrm{C}$ pendant la journée mais seulement $14^{\circ} \mathrm{C}$ les nuits et les fins de semaine. Pendant ces périodes de mise en veilleuse de la fonction chauffage, le système sera principalement utilisé dans sa fonction séparation.

Enfin ce même système de stockage d'énergie pourra être utilisé pour mettre à profit les modulations du tarif de l'électricité proposé par l'EDF selon les heures de la journée et même de la semaine.

Il conviendra alors de rechercher la meilleure combinaison entre les cycles de variation de la température extérieure (haute ou basse), les cycles de variation de la demande de chauffage et les cycles de variation du prix de l'électricité (heures «pleines» ou « creuses »).

Cette optimisation multi-critères devra être effectuée évidemment en temps réel. Elle le sera grâce à un microprocesseur intégré à l'installation.

Il sera certainement possible d'abaisser très sérieusement le prix moyen du $\mathrm{kWh}$ de chauffage grâce à une telle optimisation.

\section{Description technique de la PACCAFA.}

Les figures $2 a$ et $2 b$ montrent les schémas de circuits des fluides, respectivement pour les deux positions SÉPARATION et MÉLANGEAGE. Nous y avons repéré les états successifs de la solution par les chiffres allant de 1 à 9 et ceux du solvant par des chiffres allant de 10 à 19. La signification de ces repères est donnée dans le tableau $\mathrm{I}$.

4. 1. - Pour fixer les idées, supposons à titre d'exemple que le mélangeur fonctionne entre une température d'évaporation $T_{\text {évap }}=-5^{\circ} \mathrm{C}$ et une température de condensation $T_{\text {con }}=+55^{\circ} \mathrm{C}$.

S'il s'agissait d'une PAC à compression classique, la vapeur d'ammoniac devrait être comprimée de $3,5 \mathrm{~atm}$ à $24 \mathrm{~atm}$, comme le montre le diagramme de Oldham de la figure 3. Dans le cas extrême inverse d'une PAC à absorption classique, sans compresseur, donc isobare, le diluat sortant de l'appareil devrait avoir un titre massique $x$ en $\mathrm{NaSCN}$ égal à $63 \%$ ce qui correspond à la température d'ébullition de $55^{\circ} \mathrm{C}$ sous $3,5 \mathrm{~atm}$.

Dans le cas de la PACCAFA, on peut envisager tous les cas intermédiaires de compression-absorp- 
Tableau I.

Repères du circuit de solution

1. Diluat sortant du détendeur $\mathrm{n}^{\circ} 1$ et entrant dans le VACOSEP

2. Diluat en cours d'évaporation, en tête du VACOSEP

3. Concentrat en fin d'évaporation, sortant du VACOSEP

4. Concentrat stocké dans son réservoir

5. Concentrat sortant réchauffé de l'échangeur associé au VACOMEL

6. Concentrat entrant dans le VACOMEL

7. Diluat sortant du VACOMEL

8. Diluat sortant refroidi de l'échangeur associé au VACOMEL

9. Diluat stocké dans son réservoir.

Repères du circuit de solvant

10. Vapeur de solvant issue de la solution évaporante et en équilibre thermodynamique avec elle

11. Vapeur de solvant surpressée et surchauffée sortant du compresseur

12. Solvant sous forme vapeur sur la courbe de rosée

13. Solvant liquide sortant du condenseur

14. Solvant liquide stocké dans son réservoir

15. Solvant liquide entrant dans le détendeur $\mathrm{n}^{\circ} 2$

16. Mélange liquide-vapeur sortant du détendeur no 2 et entrant dans le VACOMEL

17. Surface de liquide en cours d'évaporation

18. Vapeur de solvant en équilibre avec le liquide précédent

19. Vapeur de solvant surpressée et surchauffée sortant du compresseur.

tion où la solution serait moins concentrée en $\mathrm{NaSCN}$ que la valeur maximale $x=63 \%$ mais où le compresseur aurait à porter la vapeur d'ammoniac de la pression $P_{18}$ à une pression $P_{19}$ inférieure à la pression maximale $P_{\text {con }}=24 \mathrm{~atm}$.

Par exemple, en comprimant de 3,5 à 11 atm, il suffira que la solution contienne $45 \%$ de NaSCN (cf. Fig. 3). A chaque valeur $P_{19}$ retenue, correspond un titre massique $x_{\mathrm{d}}$ de diluat.

D'autre part, pour réaliser ce diluat de titre $x_{\mathrm{d}}$ choisi, on peut partir d'un concentrat de titre $x_{\mathrm{c}}$ quelconque. Il suffira que $x_{\mathrm{c}}$ soit inférieure à la limite de cristallisation quand il en existe une. (Pour des raisons pra-

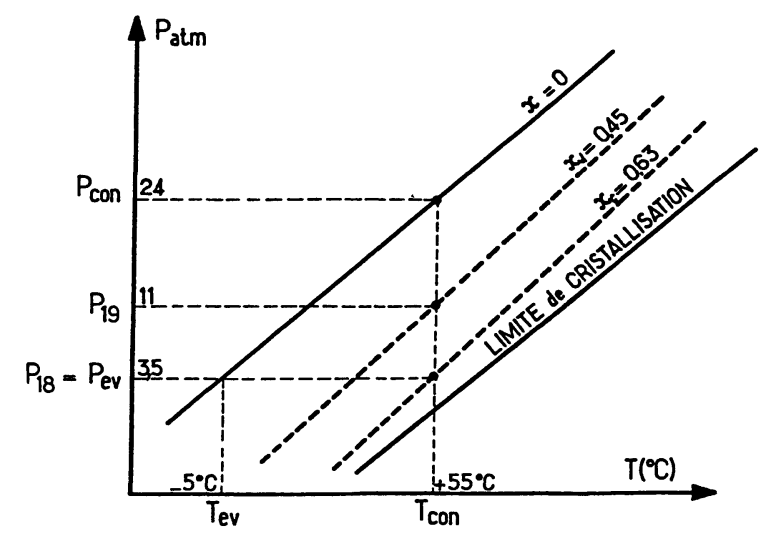

Fig. 3. - Diagramme de Oldham, sur lequel les états successifs des solutions de la PACCAFA en position VACOMEL sont représentés.

[Oldham's diagram where the different states of the solution of the CAHPAW, VACOMIX state, are pointed.] tiques, on reste généralement à 2 ou $3 \%$ en dessous de cette limite de solubilité maximale.) Dans le cas $\mathrm{NH}_{3}-\mathrm{NaSCN}$, cette valeur maximale théorique est 0,65 à température ambiante, aussi la valeur de $x_{\mathrm{c} \max }$ sera prise égale à 0,63 .

4.2. - Considérons maintenant le cas du séparateur qui fonctionne entre $T_{10}=+5^{\circ} \mathrm{C}$ et $T_{\text {con }}=55^{\circ} \mathrm{C}$.

$\mathrm{S}^{\prime}$ il s'agissait d'une PAC à compression classique, la vapeur d'ammoniac devrait être comprimée de $5,2 \mathrm{~atm}$ à $24 \mathrm{~atm}$ (cf. Fig. 4).

Dans le cas de la PACCAFA où l'évaporateur est alimenté par un diluat de titre $x_{\mathrm{d}}$ (par exemple 0,45 ) et doit former un concentrat de titre $x_{\mathrm{c}}$ (par exemple $0,63)$ tel que la pression de vapeur au-dessus de ces

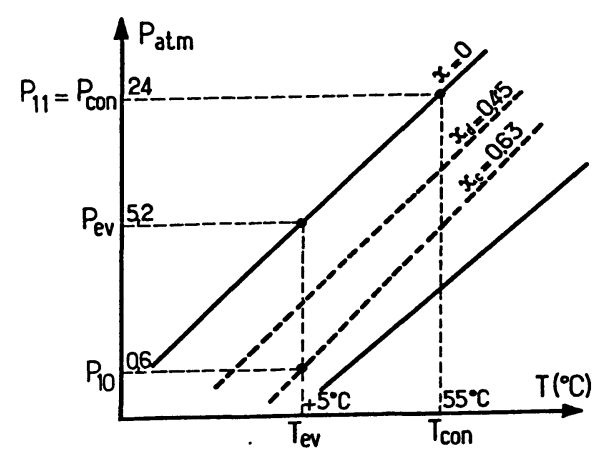

Fig. 4. - Diagramme de Oldham sur lequel les états successifs des solutions de la PACCAFA en position VACOSEP sont représentés.

[Oldham's diagram where the different states of the solution of the CAHPAW, VACOSEP state, are pointed.] 
solutions soit $P_{10}$ (avec $P_{10}<P_{\text {evap }}$; cf. Fig. 4), le compresseur devra fournir un travail supplémentaire dû à la surpression $\left(P_{\text {évap }}-P_{10}\right)$. Dans l'exemple retenu, on a $P_{10}=0,6 \mathrm{~atm}$.

4.3. - Considérons enfin l'association du séparateur et du mélangeur précédents. Supposons que nous soient imposées :

- la température d'évaporation au mélangeage, soit $T_{\text {évap }}=-5^{\circ} \mathrm{C}$

- la température d'évaporation à la séparation, soit $T_{\text {évap }}=+5^{\circ} \mathrm{C}$

- la température de condensation dans les deux cas, soit $T_{\text {con }}=+55^{\circ} \mathrm{C}$

- le débit de solvant à évaporer et condenser $\dot{M}_{\mathrm{v}}$ (fixé par la puissance de chauffe à fournir)

Il reste alors deux paramètres libres à choisir :

- ou bien les titres massiques $x_{\mathrm{d}}$ du diluat et $x_{\mathrm{c}}$ du concentrat

- ou bien les pressions de refoulement du compresseur, en séparation et en mélangeage

- ou bien une autre combinaison de deux de ces quatre paramètres.

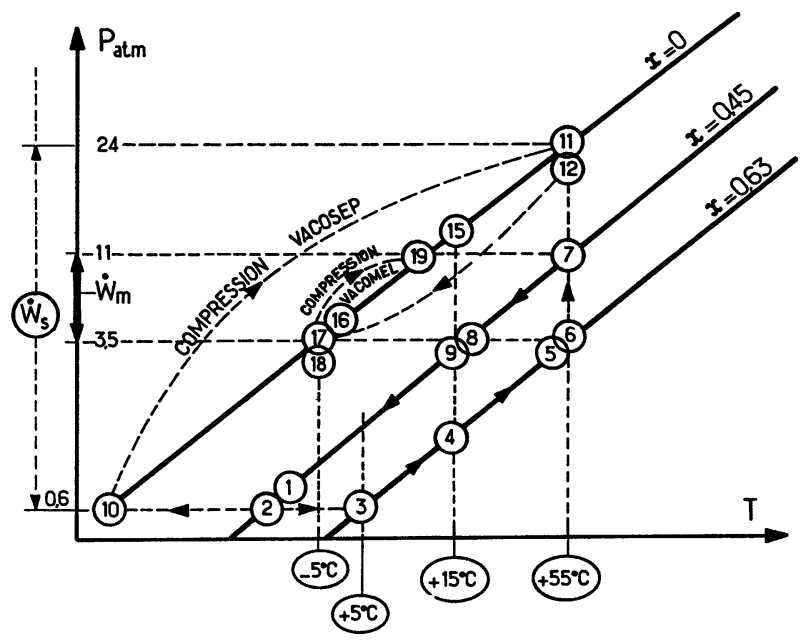

Fig. 5. - Diagramme de Oldham où les états successifs de la solution sont représentés par les chiffres de 1 à 9 conformément au tableau I. Les pressions successives (mais pas les températures) du solvant volatil sont représentées par les chiffres de 10 à 19.

[Oldham's diagram, where the different states of the solution are pointed by numbers from 1 to 9 , according to the table I. The different pressures (not the temperatures) of the volatile solvent are pointed by numbers from 10 to 19.7

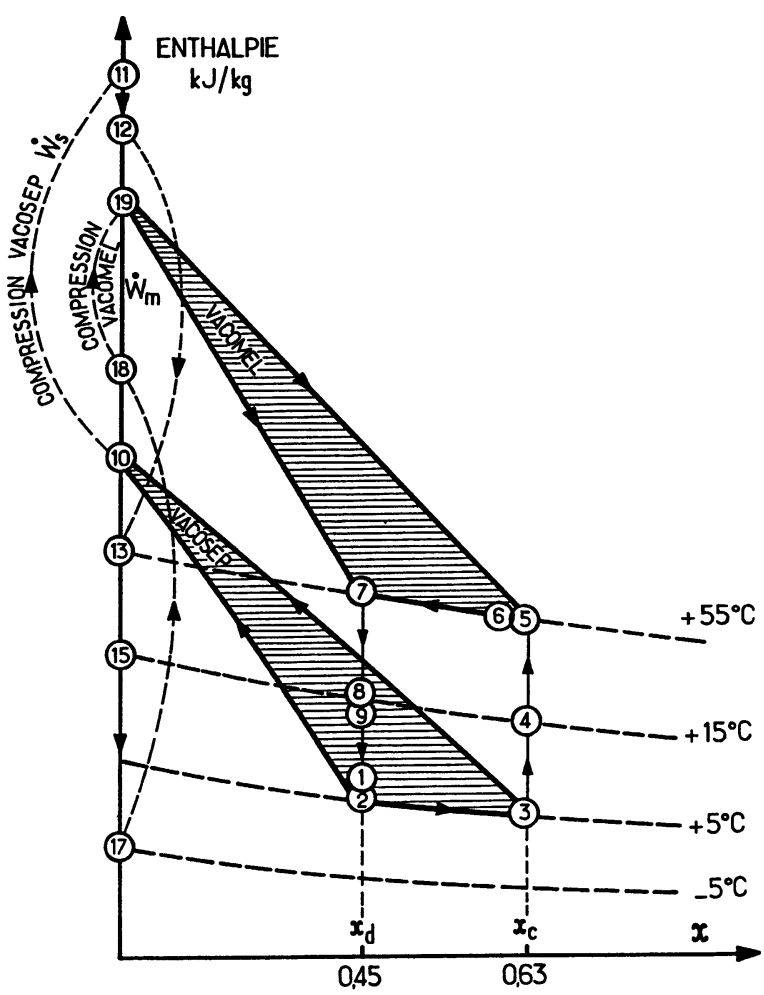

Fig. 6. - Diagramme de Ponchon où les états successifs de la solution et du solvant volatil sont représentés par les chiffres de 1 à 19.

[Ponchon's diagram, where the different states of the solution and the volatile solvent are pointed by numbers from 1 to 19.$]$

En fait nous choisirons souvent comme variables

- le titre $x_{\mathrm{d}}$ du diluat

- l'enrichissement $\Delta x=x_{\mathrm{c}}-x_{\mathrm{d}}$.

- Les figures 5 et 6 montrent respectivement les diagrammes de Oldham et de Ponchon, pour les valeurs $x_{\mathrm{c}}=0,63$ et $x_{\mathrm{d}}=0,45$. Les chiffres portés dans chaque cercle désignent les divers états successifs du solvant et de la solution, selon la nomenclature du tableau I.

\section{Expression du coefficient de performance $=$ son maximum.}

Par définition (Réf. [4]) le coefficient de performance est le rapport de la chaleur utile fournie au fluide de chauffage, à l'énergie non gratuite introduite dans le système, c'est-à-dire l'énergie mécanique fournie par le compresseur et par la pompe de circulation.

Le calcul complet des bilans enthalpique et massique conduit à l'expression suivante du COP :

$$
\mathrm{COP}=\frac{\left(x_{\mathrm{c}}-x_{\mathrm{d}}\right)\left(H_{19}+H_{11}-H_{13}\right)+x_{\mathrm{d}} H_{6}-x_{\mathrm{c}} H_{7}}{\left(x_{\mathrm{c}}-x_{\mathrm{d}}\right)\left(H_{11}-H_{10}+H_{19}-H_{18}\right)+x_{\mathrm{d}}\left(H_{6}-H_{5}\right)}
$$


On remarquera que le dénominateur de cette expression est la somme de trois termes proportionnels aux trois apports d'énergie mécanique :

$H_{11}-H_{10}$ est l'apport par le compresseur lors de la séparation

$H_{19}-H_{18}$ est l'apport par le compresseur lors du mélangeage

$H_{6}-H_{5}$ est l'apport par la pompe de circulation du concentrat.

Examinons l'influence des deux variables $x_{\mathrm{c}}$ et $x_{\mathrm{d}}$ :

- Il est facile de vérifier que, pour $x_{\mathrm{d}}$ constant, le COP est une fonction constamment croissante de $x_{\mathrm{c}}$. C'est la raison pour laquelle nous avons choisi la valeur maximale, techniquement réalisable, soit $x_{\mathrm{c}}=0,63$ comme expliqué plus haut.

- Quand $x_{\mathrm{d}}$ varie entre 0 et $x_{\mathrm{c}}$, pour la valeur constante $x_{\mathrm{c}}=0,63$, le coefficient de performance passe par un maximum comme le montre la figure 7 . Pour expliquer l'origine de ce maximum, examinons les deux cas extrêmes :

- Quand $x_{\mathrm{d}}$ tend vers zéro, le COP tend vers une valeur constante indépendante de $x_{\mathrm{c}}$, soit :

$$
\mathrm{COP}=\frac{\left(H_{19}-H_{7}\right)+\left(H_{11}-H_{13}\right)}{\left(H_{19}-H_{18}\right)+\left(H_{11}-H_{10}\right)}
$$

ce qui s'énonce :

$\mathrm{COP}=\frac{\text { gain d'enthalpie par condensation-mélangeage }}{\text { apports d'enthalpie par le compresseur }}$.

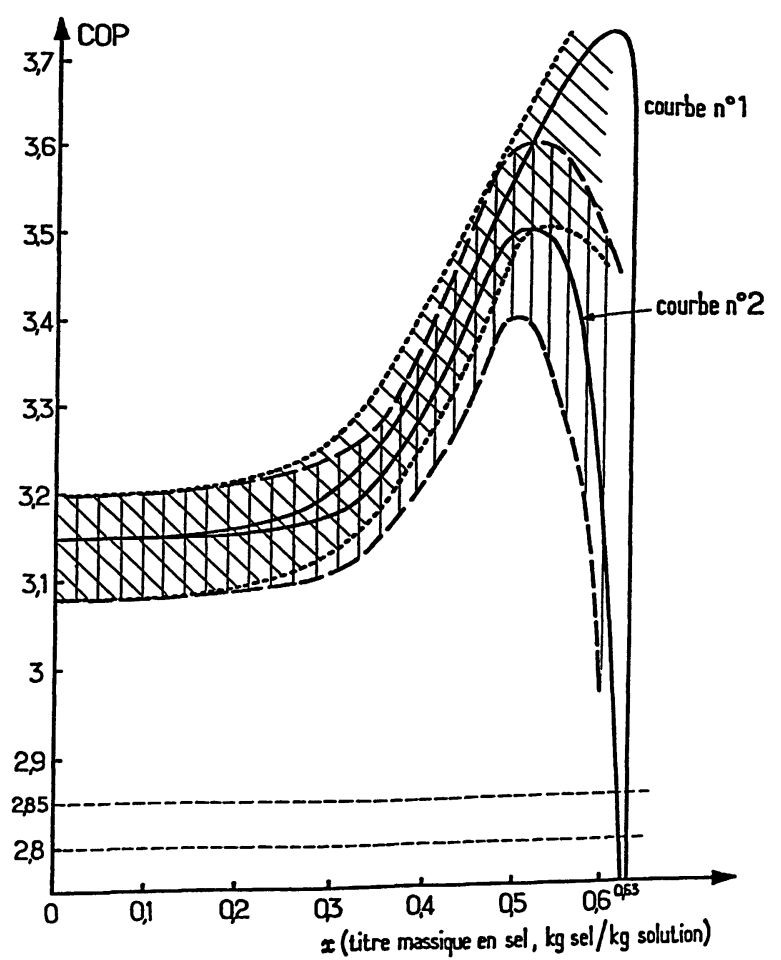

Fig. 7. - Le coefficient de performance en fonction de la concentration.

[Coefficient of performance as a function of the concentration.]
- Quand, inversement $x_{\mathrm{d}}$ tend vers $x_{\mathrm{c}}$, le numérateur tend vers zéro et de plus au dénominateur le débit de concentrat, donc aussi l'énergie fournie par la pompe de circulation, sont infiniment grands. Le COP tend donc vers zéro pour cette double raison.

- Dans l'intervalle, le COP présente un maximum dont les coordonnées dépendent des hypothèses faites sur le fonctionnement de l'échangeur de chaleur à contre-courant entre diluat et concentrat.

- Dans l'hypothèse extrême où cet échangeur est infiniment efficace, la température $T_{5}$ du concentrat sortant est alors égale à la température $T_{7}$ du diluat entrant et où tous les frottements sont nuls, le COP présente un maximum très accusé, soit :

$$
\mathrm{COP}_{\max }=3,75 \text { pour } x_{\mathrm{d}}=0,60 \text {. }
$$

- Dans l'hypothèse d'un échangeur avec pincement thermique uniforme de $5^{\circ} \mathrm{C}$ (le diluat entre à $55^{\circ} \mathrm{C}$ mais le concentrat n'en sort qu'à $50^{\circ} \mathrm{C}$ ) et en supposant encore l'absence de frottements, le COP présente un maximum moins élevé, soit :

$$
\mathrm{COP}_{\max }=3,50 \text { pour } x_{\mathrm{d}}=0,50 .
$$

En fait, vu l'incertitude sur les données enthalpiques, ces maximums ne peuvent être localisés qu'à $\pm 10 \%$ près. Les plages d'incertitude des deux courbes sont représentées sur la figure 7 .

\section{Application au chauffage d'un bâtiment.}

Supposons que la PACCAFA telle que décrite cidessus, soit utilisée pour chauffer des bâtiments dotés respectivement de trois réseaux de chauffage différents, définis comme suit, selon les conditions normalisées (Réf. [6]) :

- une "vieille " installation à radiateurs de faible surface nécessitant de l'eau à $60^{\circ} \mathrm{C}$,

- une installation plus moderne, à radiateurs plus grands, se contentant d'eau à $50^{\circ} \mathrm{C}$,

- une installation à plancher chauffant utilisant de l'eau à $35^{\circ} \mathrm{C}$.

La source de chaleur bas niveau utilisée est l'air extérieur dont la température est comprise entre $-15^{\circ} \mathrm{C}$ et $+15^{\circ} \mathrm{C}$.

- Nous admettrons, de plus, que le pincement thermique dans les évaporateurs et condenseurs est uniformément de $5^{\circ} \mathrm{C}$, soit :

$$
T_{\text {evap }}=T_{\text {ext }}-5^{\circ} \mathrm{C} \text { et } T_{\text {con }}=T_{\mathrm{u}}+5^{\circ} \mathrm{C} .
$$

Ainsi, nous avons calculé les installations de chauffage pour trois températures de condensation :

$$
T_{\text {con }}=65^{\circ} \mathrm{C}, 55^{\circ} \mathrm{C} \text { et } 40^{\circ} \mathrm{C}
$$

et pour diverses températures d'évaporation échelonnées entre : $-20^{\circ} \mathrm{C}$ et $+10^{\circ} \mathrm{C}$. 
Les résultats de ces calculs sont présentés sur les figures 8,9 et 10. Dans chaque cas, le COP calculé par la relation (1) est porté en fonction de la température extérieure, lors du fonctionnement en séparateur, pour cinq valeurs du paramètre température extérieure, lors du fonctionnement en mélangeur. La courbe en trait gras correspond au cas où la PACCAFA fonctionne avec la même température extérieure, aussi bien en séparateur qu'en mélangeur.

Sur chaque figure nous avons également porté deux autres courbes :

- la valeur théorique maximale donnée par la relation

$$
\mathrm{COP}_{\text {max }}=\frac{T_{\text {con }}}{T_{\text {con }}-T_{\text {évap }}}
$$

- la valeur théorique d'une pompe à chaleur à compression, opérant avec de l'ammoniac pur et sans aucune irréversibilité soit, par lecture sur le diagramme de Mollier :

$$
\operatorname{COP}_{\mathrm{th}}=\frac{\Delta H_{\text {condensation }}}{\Delta H_{\text {compression isentrope }}}
$$

\section{Commentaires et conclusions.}

L'examen des figures 8, 9 et 10 appelle les commentaires suivants :

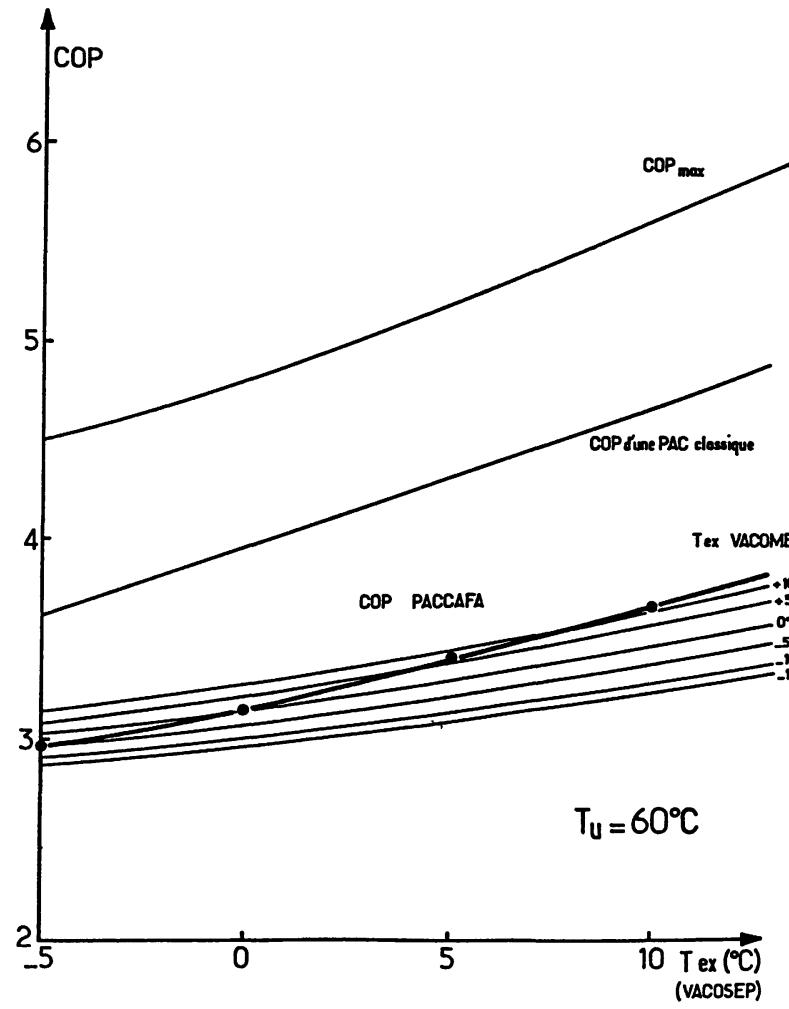

Fig. 8. - Coefficient de performance en fonction de la température extérieure pour une température utile $=60^{\circ} \mathrm{C}$.

[Coefficient of performance as a function of outside temperature for a useful temperature $=60{ }^{\circ} \mathrm{C}$.]
1. Pour un couple de températures extérieures données $\left(T_{1}, T_{2}\right)$ avec $T_{1}>T_{2}$, on observe que le COP est plus grand quand le séparateur opère avec $T_{1}$ et le mélangeur avec $T_{2}$, plutôt que dans le cas inverse. Ceci confirme bien notre choix de faire fonctionner l'appareil en mélangeur lorsqu'il fait le plus froid et en séparateur quand le temps devient plus clément.

2. Le COP d'une PAC classique, tout en restant supérieur au COP d'une PACCAFA, opérant toujours aux mêmes températures, se rapproche de celui-ci lorsque $T_{\mathrm{u}}$ croît. En effet, pour une température extérieure donnée, le COP d'une PAC classique est une fonction très croissante de $T_{\mathrm{u}}$, alors que le COP de la PACCAFA reste sensiblement du même ordre de grandeur, tout en diminuant lorsque $T_{\mathrm{u}}$ croît.

Le COP d'une PACCAFA et le COP d'une PAC classique seront donc d'un même ordre de grandeur pour des températures utiles de l'ordre de $70-80^{\circ} \mathrm{C}$.

3. De toute façon, cette comparaison ne porte que sur des maxima théoriques, c'est-à-dire en négligeant toutes les irréversibilités. Cette étude devra donc être refaite ultérieurement, en comparant les valeurs réelles des coefficients de performance.

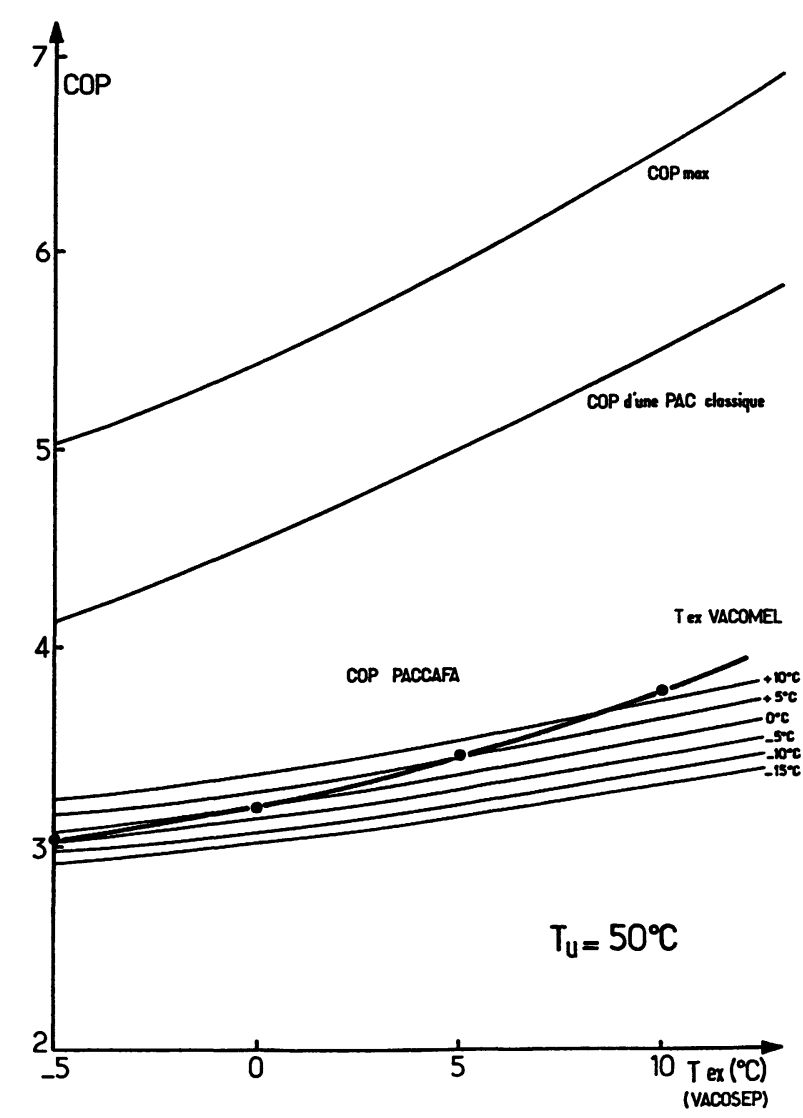

Fig. 9. - Coefficient de performance en fonction de la température extérieure pour $T$ utile $=50^{\circ} \mathrm{C}$.

[Coefficient of performance as a function of outside temperature for a useful temperature $=50^{\circ} \mathrm{C}$.] 


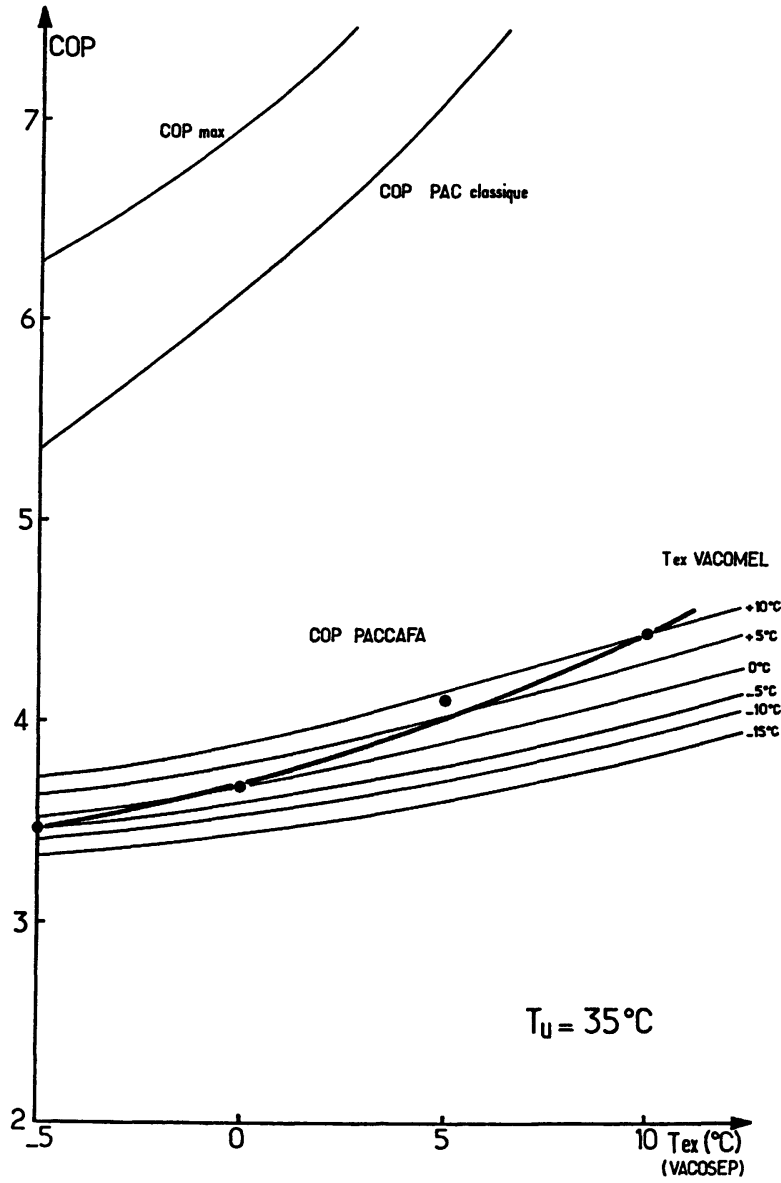

Fig. 10. - Coefficient de performance en fonction de la température extérieure pour $T$ utile $=35^{\circ} \mathrm{C}$.

[Coefficient of performance as a function of outside temperature for a useful temperature $=35^{\circ} \mathrm{C}$.]
Nous retiendrons seulement ici que pour des systèmes de chauffage classique par radiateurs à $60^{\circ} \mathrm{C}$ ou $70-80^{\circ} \mathrm{C}$, la PACCAFA a un COP théorique à peine inférieur ou du même ordre de grandeur que celui d'une PAC à compression classique.

Rappelons d'autre part que la PACCAFA présente des avantages décisifs :

- La chaudière de combustion, d'appoint pour périodes froides, peut être supprimée.

- Le compresseur travaille à puissance quasi constante, donc avec un meilleur rendement, optimisé.

- L'existence du stockage interne, apporte une souplesse de fonctionnement permettant une optimisation multi-critères, tenant compte à la fois des fluctuations de la température extérieure, de la demande des utilisateurs et des tarifs de l'électricité (heures pleines, heures creuses).

- Les seuls équipements supplémentaires par rapport à une PAC à compression classique sont : un échangeur liquide/liquide (entre concentrat et diluat) et les trois réservoirs de stockage.

De ces facteurs il résulte que le coût d'investissement d'une PACCAFA sera très probablement nettement inférieur à celui d'une PAC à compression et de sa chaudière d'appoint. Il est probable que ces réductions du coût d'investissement et du coût de l'énergie électrique consommée, compenseront largement la faible réduction du Coefficient de Performance. Nous consacrerons une publication ultérieure à l'évaluation détaillée de la rentabilité économique de la PACCAFA.

\section{Bibliographie}

[1] DieTrich, E. et LE GofF, P., Un nouveau type de pompe à chaleur associant une compression, une absorption et des réservoirs de stockage. - Congrès Institut International du Froid, Commission E2, Trondheim, Norvège, juin 1985.

[2] Brevets CNRS-ANVAR (Inv. P. Le Goff) no 80.17676, $\mathrm{n}^{\circ} 81.13846, \mathrm{n}^{\circ} 81.15263$ et $\mathrm{n}^{\circ} 82.07510$.

[3] DieTRICH, E., Thèse Docteur-Ingénieur, I.N.P.L., juillet 1984 .

[4] Dietrich, E., Le Goff, P., Barkaoui, M., Entropie 118 (1984) 8-23.
[5] Dietrich, E., Le Goff, P., Le Goff, H., Barkaoui, M., Ramadane, A., TraP, J. C., Une nouvelle technologie de la pompe à chaleur à absorption multiétagée, avec stockage incorporé de l'exergie. Rev. Géné. Thermique 281 (1985) 537 à 545.

[6] CECAL, groupe d'études à long terme sur le chauffage. Le chauffage des habitations en France en l'an 2000 - évaluations et perspectives (Editions Européennes Thermique et Industrie (E.E.T.I.)) (1981). 